ALEA, Lat. Am. J. Probab. Math. Stat. 16, 439-461 (2019)

DOI: 10.30757/ALEA.v16-17

\title{
Long-memory Gaussian processes governed by generalized Fokker-Planck equations
}

\author{
Luisa Beghin \\ Department of Statistical Sciences, Sapienza - University of Rome \\ E-mail address: luisa.beghin@uniroma1.it
}

\begin{abstract}
It is well-known that the transition function of the Ornstein-Uhlenbeck process solves the Fokker-Planck equation. This standard setting has been recently generalized in different directions, for example, by considering the so-called $\alpha$-stable driven Ornstein-Uhlenbeck, or by time-changing the original process with an inverse stable subordinator. In both cases, the corresponding partial differential equations involve fractional derivatives (of Riesz and Riemann-Liouville types, respectively) and the solution is not Gaussian. We consider here a new model, which cannot be expressed by a random time-change of the original process: we start by a Fokker-Planck equation (in Fourier space) with the time-derivative replaced by a new fractional differential operator. The resulting process is Gaussian and, in the stationary case, exhibits long-range dependence. Moreover, we consider further extensions, by means of the so-called convolution-type derivative.
\end{abstract}

\section{Introduction and notation}

The Ornstein-Uhlenbeck process has been widely studied and applied in many different fields, mainly in finance and physics. Indeed, its mean-reverting property is useful, in particular, in the financial data description, since many economic quantities (such as interest rates) appear to be pulled back to some average value, in the long-term period. Moreover, it is Gaussian, Markovian and has a stationary version, which is the only nontrivial process satisfying all the previous conditions.

It is well-known that the density of the Ornstein-Uhlenbeck (hereafter OU) process satisfies the so-called Fokker-Planck (FP) equation, i.e.

$$
\frac{\partial}{\partial t} u(x, t)=\eta \frac{\partial}{\partial x}[x u(x, t)]+D \frac{\partial^{2}}{\partial x^{2}} u(x, t), \quad x \in \mathbb{R}, t \geq 0, D, \eta>0,
$$

with the initial condition $u(x, 0)=\delta(x)$ and the boundary condition $\lim _{|x| \rightarrow+\infty} x u(x, t)=0$, for any $t \geq 0$ (see, for example, Pavliotis, 2014, p.95).

Received by the editors October 24th, 2018; accepted February 27th, 2019.

2010 Mathematics Subject Classification. 60G15, 60G22, 34A08, 33C60, 26 A33.

Key words and phrases. Fractional Fokker-Planck equation; Long-range dependence; Hfunctions; Convolution-type derivative. 
Fractional extensions of the previous equation have been proposed by many authors, in different directions: in particular, when a fractional Riemann-Liouville derivative (with respect to time) is introduced in the right-hand side, an anomalous diffusion is obtained, which can be described as a random time-change of the OU process by means of the inverse of an independent stable subordinator (see Magdziarz et al., 2007 and Magdziarz, 2009). In Janczura et al. (2011) this model is compared to the OU process driven by the $\alpha$-stable Lévy motion (which is governed by equation (1.1) with the second order derivative replaced by the Riesz fractional operator). Later, in Gajda and Wyłomańska (2015), the model has been generalized to the case where the time-derivative in (1.1) is replaced by a more general integro-differential operator with memory kernel; as a consequence, the time-change is by means of the inverse of a general Lévy subordinator.

Another approach to fractional OU processes has been proposed in Magdziarz (2008) where the solution of the $\alpha$-stable Langevin equation (the so-called $\alpha$-stable OU process) is extended to the fractional case, tracing the transition form Brownian motion to fractional Brownian motion. The resulting processes exhibit long-range dependence, which, due to the lack of finite second moments, must be measured by codifferences.

Other non-Gaussian OU-type processes have been obtained by applying the Lamperti transform to the fractional Brownian motion (see Cheridito et al., 2003) and they are proved to display short-range dependence when the Hurst parameter $H$ is greater than $1 / 2$ (on the contrary to that obtained from the Langevin equation with fractional noise). Also the extension of OU defined in Kaarakka and Salminen (2011), by the Doob's transform of fractional Brownian motion, has covariance function decaying exponentially for all values of $H$.

The class of time-fractional Pearson diffusions have also been studied in Leonenko et al. (2013) and the fractional OU process is treated there as a particular case. All these models are non-Markovian, as described in Mura et al. (2008), and nonGaussian. Finally, we recall that fractional diffusion equations with logarithmictype differential operators have been studied in Beghin (2018) and, as in the cases cited above, a random time change (or subordination) is proved to hold for the corresponding process.

We present here different models, alternative to all those described above, which generalize the OU process in a fractional (and non-fractional) sense, but preserve the Gaussianity of its distribution. Moreover, contrary to the $\alpha$-stable OU and fractional $\alpha$-stable OU processes (in Magdziarz (2008)), they have finite moments and, indeed, they are characterized by means of their covariance function (since they are Gaussian).

As we will see, in our case, no random time change is entailed by the introduction of the fractional operator, being in Fourier space. This is the most important feature of our results and shows that correspondence between partial differential equations with fractional (or convolution-type derivatives) and subordination (or time-change) does not always come true.

The first model is called time-changed OU, since we prove that introducing, in the Fourier transform of (1.1), a fractional operator entails a deterministic timechange in the original process, expressed in terms of the Mittag-Leffler function. Hence the process is still Gaussian, mean-reverting and Markovian, even though, in the limit (for $t \rightarrow \infty$ ), it does not converge to its stationary counterpart (as 
in the standard case), but to a random walk. In order to correct this anomalous asymptotic behavior, we define a second fractional OU process, whose (Gaussian) one-dimensional density satisfies the same fractional FP equation, but is not Markovian and has a stationary counterpart, in the limit. We show that it presents long-range dependence, both in the covariance and in the spectral density sense. We remark that, in all these cases, when the fractional parameter tends to one, we recover the classical OU model.

As a motivation of the results described above, we show that the solution of the fractional FP equation coincides with the one-dimensional distribution of the limiting process obtained from a Poisson shot-noise, with power-law decaying response function, when the frequency of the shocks increases and their amplitude tends to zero.

Further extensions of the previous results are obtained, in the last section, by generalizing the fractional operator adopted, by means of the convolution-type derivative (see Gajda and Wyłomańska, 2015 and Toaldo, 2015 among the others) and solving the corresponding FP equation (in Fourier space). The solution coincides with the characteristic function of a generalized OU process, which is then defined through the inverse of a general subordinator.

We now introduce some useful definitions and notation.

First of all, let $f: \mathbb{R} \rightarrow \mathbb{R}$ be an integrable function belonging to the Schwartz space $\mathcal{S}(\mathbb{R})$ of rapidly decreasing functions; then we denote by $\mathcal{F}(f ; \xi):=$ $\int_{-\infty}^{+\infty} e^{i \xi x} f(x) d x=\widehat{f}(\xi)$ the Fourier transform and by $\mathcal{F}^{-1}(f ; x):=$ $\frac{1}{2 \pi} \int_{-\infty}^{+\infty} e^{-i \xi x} \widehat{f}(\xi) d \xi$ its inverse. Moreover, for $s>0$, we denote the Laplace transform of $f$, as $\mathcal{L}(f ; s):=\int_{0}^{+\infty} e^{-s x} f(x) d x=\widetilde{f}(s)$.

Let $\alpha>0, m=\lfloor\alpha\rfloor+1$ and assume that $u:[a, b] \rightarrow \mathbb{R}, b>a$, is an absolutely continuous function, with absolutely continuous derivatives up to order $m$ on $[a, b]$, then, for $x \in[a, b]$,

$$
D_{x}^{\alpha} u(x):=\left\{\begin{array}{l}
\frac{1}{\Gamma(m-\alpha)} \int_{a}^{x} \frac{1}{(x-s)^{\alpha-m+1}} \frac{d^{m}}{d s^{m}} u(s) d s, \quad \alpha \notin \mathbb{N}_{0} \\
\frac{d^{m}}{d x^{m}}, \quad \alpha=m \in \mathbb{N}_{0}
\end{array}\right.
$$

is the Caputo fractional derivative of order $\alpha$ (see Kilbas et al., 2006, p.92). We define here the new following fractional operator.

Definition 1.1. Let $\alpha \geq 0$ and $m=\lfloor\alpha\rfloor+1$. Let $u(\cdot):[0, b], b>0$, be a positive function, with absolutely continuous derivatives up to order $m$ on $[0, b]$, then

$$
\mathcal{L}_{x}^{\alpha} u(x):=u(x) D_{x}^{\alpha} \log u(x), \quad x \in \mathbb{R},
$$

where $D_{x}^{\alpha}$ is the Caputo fractional derivative of order $\alpha$.

It is easy to check that, for $\alpha=1$, the operator defined in (1.3) coincides with the first-order derivative, i.e.

$$
\mathcal{L}_{x}^{1} u(x)=\frac{d}{d x} u(x) .
$$

The previous definition can be generalized by considering the convolution-type derivative (see Toaldo, 2015), which is defined by means of the subordinators' theory. Let $g:(0,+\infty) \rightarrow \mathbb{R}$ be a Bernstein function, i.e. let $g$ be non-negative, 
infinitely differentiable and such that, for any $x \in(0,+\infty)$,

$$
(-1)^{n} \frac{d^{n}}{d x^{n}} g(x) \leq 0, \quad \text { for any } n \in \mathbb{N} .
$$

A function $g$ is a Bernstein function if and only if it admits the following representation

$$
g(x)=a+b x+\int_{0}^{+\infty}\left(1-e^{-s x}\right) \bar{\nu}(d s)
$$

where $\bar{\nu}$ is the Lévy measure and $(a, b, \bar{\nu})$ is called the Lévy triplet of $g$. Then a subordinator is the stochastic process with non-decreasing paths $\mathcal{A}^{g}:=\left\{\mathcal{A}^{g}(t), t \geq 0\right\}$, such that

$$
\mathbb{E} e^{-s \mathcal{A}^{g}(t)}=e^{-g(s) t},
$$

(see Applebaum, 2009 and Sato, 1999 for the theory of Lévy processes and subordinators). Let moreover $L^{g}(t), t \geq 0$, be the inverse of $\mathcal{A}^{g}$, i.e.

$$
L^{g}(t)=\inf \left\{s \geq 0: \mathcal{A}^{g}(s)>t\right\}, \quad t>0
$$

and $l_{g}(x, t)=\operatorname{Pr}\left\{L^{g}(t) \in d x\right\}$ be its transition density.

We recall the definition of the convolution-type derivative on the positive halfaxes, in the sense of Caputo (see Toaldo, 2015, Def.2.4, for $b=0$ ):

$$
D_{t}^{g} u(t):=\int_{0}^{t} \frac{d}{d s} u(t-s) \nu(s) d s, \quad t>0,
$$

where $\nu$ is the tail of the Lévy measure $\bar{\nu}$, i.e. $\nu(s) d s=a+\bar{\nu}(s, \infty) d s$. For $g(s)=s^{\alpha}$ with $\alpha \in(0,1)$, the process $\mathcal{A}^{g}$ coincides with the $\alpha$-stable subordinator with $\mathbb{E} e^{-s \mathcal{A}^{g}(t)}=e^{-s^{\alpha} t}$ (whose Lévy measure is $\bar{\nu}(s)=s^{-\alpha-1} / \Gamma(m-\alpha)$ ) and formula (1.5) reduces to the Caputo derivative (1.2), for $m=1$.

The Laplace transform of $D_{t}^{g}$ is given by

$$
\int_{0}^{+\infty} e^{-s t} D_{t}^{g} u(t) d t=g(s) \widetilde{u}(s)-\frac{g(s)}{s} u(0), \quad \mathcal{R}(s)>s_{0},
$$

(see Toaldo, 2015, Lemma 2.5).

Let $E_{\beta, \gamma}(x)$ denote the generalized Mittag-Leffler function defined as

$$
E_{\beta, \gamma}(x)=\sum_{j=0}^{\infty} \frac{x^{\beta j}}{\Gamma(\beta j+\gamma)}, \quad x, \beta, \gamma \in \mathbb{C}, \operatorname{Re}(\beta), \operatorname{Re}(\gamma)>0,
$$

(see Haubold et al., 2011), then we recall the well-known formula of its Laplace transform (see Kilbas et al., 2006, formula (1.9.13), for $\rho=1$ ), i.e.

$$
\mathcal{L}\left\{x^{\gamma-1} E_{\beta, \gamma}\left(A x^{\beta}\right) ; s\right\}=\frac{s^{\beta-\gamma}}{s^{\beta}-A},
$$

with $\operatorname{Re}(\beta), \operatorname{Re}(\gamma)>0, A \in \mathbb{R}$ and $s>|A|^{1 / \operatorname{Re}(\beta)}$.

We recall also the well-known (power-law) asymptotic behavior of the MittagLeffler function (see Kilbas et al., 2006, formula (1.8.11)), for $|z| \rightarrow+\infty$, i.e.

$$
E_{\beta, \gamma}(z)=-\sum_{k=1}^{n} \frac{z^{-k}}{\Gamma(\gamma-\beta k)}+O\left(z^{-n-1}\right), \quad n \in \mathbb{N},
$$

which holds for $0<\beta<2, \mu \leq \arg (z) \leq \pi$, where $\pi \beta / 2<\mu<\min \{\pi, \pi \beta\}$. 
We will also make use of the property of the function (1.7) of being completely monotone (CM), which is proved in Schneider (1996), for a negative argument and $\beta \in(0,1], \gamma \geq \beta$. By definition, a function $f(x)$ is $\mathrm{CM}$ if and only if it is infinitely differentiable and $(-1)^{n} \frac{d^{n}}{d x^{n}} f(x) \geq 0$, for any $n=0,1, \ldots$

Finally we recall the definition of Fox's H-functions, which will by applied in the next sections. Let $H_{p, q}^{m, n}$ denote the H-function defined as (see Mathai et al., 2010 p.13):

$$
\begin{aligned}
& H_{p, q}^{m, n}\left[z \mid \begin{array}{lll}
\left(a_{1}, A_{1}\right) & \ldots & \left(a_{p}, A_{p}\right) \\
\left(b_{1}, B_{1}\right) & \ldots & \left(b_{q}, B_{q}\right)
\end{array}\right] \\
& =\frac{1}{2 \pi i} \int_{\mathcal{L}} \frac{\left\{\prod_{j=1}^{m} \Gamma\left(b_{j}+B_{j} s\right)\right\}\left\{\prod_{j=1}^{n} \Gamma\left(1-a_{j}-A_{j} s\right)\right\} z^{-s} d s}{\left\{\prod_{j=m+1}^{q} \Gamma\left(1-b_{j}-B_{j} s\right)\right\}\left\{\prod_{j=n+1}^{p} \Gamma\left(a_{j}+A_{j} s\right)\right\}},
\end{aligned}
$$

with $z \neq 0, m, n, p, q \in \mathbb{N}_{0}$, for $1 \leq m \leq q, 0 \leq n \leq p, a_{j}, b_{j} \in \mathbb{R}, A_{j}, B_{j} \in \mathbb{R}^{+}$, for $i=1, \ldots, p, j=1, \ldots, q$ and $\mathcal{L}$ is a contour such that the following condition is satisfied

$$
A_{\lambda}\left(b_{j}+\alpha\right) \neq B_{j}\left(a_{\lambda}-k-1\right), \quad j=1, \ldots, m, \lambda=1, \ldots, n, \alpha, k=0,1, \ldots
$$

\section{Auxiliary results}

We consider the fractional diffusion equation obtained by replacing the time derivative with the pseudo-differential operator $\mathcal{L}_{t}^{\alpha}$ defined in Def.1.1 in the standard heat equation (in the Fourier space), i.e.

$$
\frac{\partial}{\partial t} \widehat{u}(\xi, t)=-\frac{\xi^{2}}{2} \widehat{u}(\xi, t) \quad \xi \in \mathbb{R}, t \geq 0, \quad \text { with } \widehat{u}(\xi, 0)=1 .
$$

Lemma 2.1. Let $\mathcal{L}_{t}^{\alpha}$ be the operator defined in Def.1.1, then the solution to the following equation

$$
\mathcal{L}_{t}^{\alpha} \widehat{u}(\xi, t)=-\frac{\Gamma(\alpha+1) \xi^{2}}{2} \widehat{u}(\xi, t) \quad \xi \in \mathbb{R}, t \geq 0, \alpha \in(0,1],
$$

is given by the characteristic function of a standard Brownian motion $W \doteq W(t)$, $t \geq 0$, time-changed by $\mathcal{T}(t)=t^{\alpha}$, for any $t \geq 0$, i.e. $W_{\alpha}(t):=W\left(t^{\alpha}\right)$.

Proof: By considering (1.3), we get from (2.1) that

$$
D_{t}^{\alpha} \log \widehat{u}(\xi, t)=-\frac{\Gamma(\alpha+1) \xi^{2}}{2},
$$

with $\log \widehat{u}(\xi, 0)=0$. Then we take the Laplace transform and obtain, by taking into account formula (2.4.62) in Kilbas et al. (2006) (with the initial condition),

$$
s^{\alpha} \int_{0}^{+\infty} e^{-s t} \log \widehat{u}(\xi, t) d t-s^{\alpha-1} \log \widehat{u}(\xi, 0)=-\frac{\Gamma(\alpha+1) \xi^{2}}{2 s}
$$

and then

$$
\log \widehat{u}(\xi, t)=-\frac{\xi^{2} t^{\alpha}}{2}
$$

which gives $\widehat{u}(\xi, t)=e^{-\xi^{2} t^{\alpha} / 2}, \xi \in \mathbb{R}, t \geq 0$. 
We remark that the introduction of the operator $\mathcal{L}_{t}^{\alpha}$ entails a deterministic time change in the corresponding process, contrary to what happens when substituting the time-derivative with the Caputo (or the Riemann-Liouville) fractional derivative in the heat equation. In the latter case, a random time-change is produced, by means of an independent inverse stable subordinator (see, e.g., Meerschaert and Sikorskii, 2012, p.42).

In this case, since the function $\mathcal{T}_{\alpha}(\cdot): \mathbb{R}^{+} \rightarrow \mathbb{R}^{+}$is continuous, increasing and such that $\mathcal{T}_{\alpha}(0)=0$, as a consequence, $W_{\alpha}(t)$ is an additive process (see Cont and Tankov, 2004, p. 469 and Sato, 1999, p.3), i.e. the Brownian motion (which is a Lévy process) looses the stationarity of its increments, by effect of the time-change, but the latter are still independent. Indeed, we get, for $0 \leq t_{1} \leq t_{2}$,

$$
\mathbb{E}\left(W_{\alpha}\left(t_{2}\right)-W_{\alpha}\left(t_{1}\right)\right)^{2}=t_{2}^{\alpha}-t_{1}^{\alpha} \neq\left(t_{2}-t_{1}\right)^{\alpha}=\mathbb{E}\left(W_{\alpha}\left(t_{2}-t_{1}\right)\right)^{2},
$$

unless $\alpha=1$. Finally, we notice that $W_{\alpha}$ is a self-similar process with Hurst exponent equal to $\alpha / 2$, since, for any $c>0$,

$$
c^{\alpha / 2} W_{\alpha}(t) \stackrel{d}{=} W_{\alpha}(c t), \quad \text { for any } t \geq 0 .
$$

The process $W_{\alpha}$ is already known in the literature as "scaled Brownian motion" (see Lim and Muniandy, 2002, Jeon et al., 2014), and is used to model anomalous diffusion (i.e. diffusion with mean-squared displacement $\left\langle W_{\alpha}(t)^{2}\right\rangle \sim t^{\alpha}$, for $\alpha \in$ $(0,2))$. As we will see in the next section, the introduction of the operator $\mathcal{L}_{t}^{\alpha}$ in the Fokker-Planck equation (in Fourier space) will entail a deterministic timechange in the corresponding OU process, which, instead of $\mathcal{T}_{\alpha}(t)=t^{\alpha}$, is expressed by means of the Mittag-Leffler process (see formula (3.3) below).

As a motivation for our analysis, we introduce the following Poisson shot-noise process and study its asymptotic behavior.

Definition 2.2. (Power-law shot-noise) Let $\{N(t), t \geq 0\}$ be an homogeneous Poisson process, with rate $\lambda>0$, and let $T_{j}$ be the occurring time of its $j$-th event, for $j=1,2, \ldots$, then we define the generalized shot-noise process as

$$
S_{\alpha}(t):=\sum_{j=1}^{N(t)} h\left(t-T_{j}\right),
$$

with $h(u)=\sqrt{u^{\alpha-1} E_{\alpha, \alpha}\left(-\gamma(2 u)^{\alpha}\right)} 1_{\{u>0\}}, \alpha \in(0,1]$.

Remark 2.3. By considering formula (16.85) in Papoulis (1991), p.632, for $h(\cdot)$ given above, it can be easily checked that the joint cumulant generating function of $S_{\alpha}$ is equal to

$$
C_{\alpha}(\underline{\theta}, \underline{t}):=\log \mathbb{E} e^{\theta_{1} S_{\alpha}\left(t_{1}\right)+\ldots+\theta_{k} S_{\alpha}\left(t_{k}\right)}=\lambda \int_{-\infty}^{+\infty}\left[e^{\theta_{1} h\left(t_{1}-u\right)+\ldots+\theta_{k} h\left(t_{k}-u\right)}-1\right] d u,
$$

where $\underline{\theta}:=\left(\theta_{1}, \ldots, \theta_{k}\right)$ and $\underline{t}:=\left(t_{1}, . ., t_{k}\right)$, for any $k \in \mathbb{N}$. Moreover, by applying formula (1.9), we can see that $h(u) \simeq k u^{-\frac{\alpha}{2}-\frac{1}{2}}$, for $u \rightarrow \infty$ and for a constant $k>0$. The function $h$ is decreasing, since both terms under square root in its expression are $\mathrm{CM}$, for $\alpha \in(0,1]$, and thus the same is true for their product; the square root does not affect the decreasing behavior. Therefore the previous model is well suited to the case where the values of the shocks (occurring at Poisson times) decrease in time with a power law (thus slower than in the, usual, exponential case). 
We now study the limiting behavior of $S_{\alpha}:=\left\{S_{\alpha}(t), t \geq 0\right\}$, after centering (2.3) (subtracting its mean $\mu_{n}$ ) and by a proper rescaling of the amplitude of the shocks and their frequency.

Lemma 2.4. Let $\left\{N_{n}(t), t \geq 0\right\}$ be an homogeneous Poisson process, with rate $\lambda=$ $n \lambda_{0}$, for $\lambda_{0}>0$, and let $h(u)=\frac{1}{\sqrt{n}} h_{0}(u)$, with $h_{0}(u)=$ $\sqrt{\left(u+\xi_{0}\right)^{\alpha-1} E_{\alpha, \alpha}\left(-\gamma 2^{\alpha}\left(u+\xi_{0}\right)^{\alpha}\right)} 1_{\{u>0\}}$, for $n \in \mathbb{N}$, some $\xi_{0}>0$. Under these assumptions, the process

$$
U_{\alpha, n}(t):=\frac{1}{\sqrt{n}}\left[\sum_{j=1}^{N_{n}(t)} h_{0}\left(t-T_{j}\right)-\mu_{n}(t)\right],
$$

converges weakly, for $n \rightarrow+\infty$, to a Gaussian process $U_{\alpha}:=\left\{U_{\alpha}(t), t \geq 0\right\}$ with joint cumulant generating function

$$
\log \mathbb{E} e^{\theta_{1} U_{\alpha}\left(t_{1}\right)+\ldots+\theta_{k} U_{\alpha}\left(t_{k}\right)}=\frac{\lambda_{0}}{2} \int_{-\infty}^{+\infty}\left[\theta_{1} h_{0}\left(t_{1}-u\right)+\ldots+\theta_{k} h_{0}\left(t_{k}-u\right)\right]^{2} d u .
$$

Proof: The convergence of the finite dimensional distributions can be easily derived, following Papoulis (1991), p.633, by the Taylor's series expansion of (2.4). See also Papoulis (1971) and Heinrich and Schmidt (1985). Then we prove tightness of the sequence, by proving the following sufficient conditions (see Revuz and Yor, 1999, p.516):

$$
P\left(\left|U_{\alpha, n}(0)\right|>A\right) \leq \varepsilon
$$

for any $n \geq n_{0}, \varepsilon>0$ and for some $n_{0}, A$,

$$
\delta^{-1} P\left(\sup _{|t-s|<\delta}\left|U_{\alpha, n}(t)-U_{\alpha, n}(s)\right| \geq \eta\right) \leq \varepsilon
$$

for $s<t$ and for some $\delta \in(0,1)$. The first condition is trivially satisfied since the process starts a.s. from zero. The check that condition (2.7) holds is carried out by applying the Doob submartingale's inequality (see Revuz and Yor, 1999, p.52), i.e.

$$
\eta^{p} P\left(\sup _{t} X_{t} \geq \eta\right) \leq \sup _{t} \mathbb{E} X_{t}^{p},
$$

for $p \geq 1$ and for a positive submartingale $X_{t}$. We choose $p=4$ and show that the difference process in (2.7) is a positive submartingale, since its fourth moment is increasing in $t$, for fixed $t-s$. Indeed, we can write (by denoting $\bar{h}_{0}(\cdot)$ the centered addends)

$$
\begin{aligned}
& U_{\alpha, n}(t)-U_{\alpha, n}(s)=\frac{1}{\sqrt{n}}\left[\sum_{j=1}^{N_{n}(t)} h_{0}\left(t-T_{j}\right)-\mu_{n}(t)-\sum_{j=1}^{N_{n}(s)} h_{0}\left(s-T_{j}\right)+\mu_{n}(s)\right] \\
& =\frac{1}{\sqrt{n}}\left[\sum_{j=1}^{N_{n}(s)} \bar{h}_{0}\left(t-T_{j}\right)+\sum_{j=N_{n}(s)+1}^{N_{n}(t)} \bar{h}_{0}\left(t-T_{j}\right)-\sum_{j=1}^{N_{n}(s)} \bar{h}_{0}\left(s-T_{j}\right)\right] \\
& =\frac{1}{\sqrt{n}}\left[\sum_{j=N_{n}(s)+1}^{N_{n}(t)} \bar{h}_{0}\left(t-T_{j}\right)-\sum_{j=1}^{N_{n}(s)}\left[\bar{h}_{0}\left(s-T_{j}\right)-\bar{h}_{0}\left(t-T_{j}\right)\right]\right] \\
& =U_{\alpha, n}^{*}(t-s)-W_{\alpha, n}(s ; t)
\end{aligned}
$$


where $U_{\alpha, n}^{*}$ is a new, centered, shot-noise (starting from $s$ and evaluated in $t$ ), which depends only on the Poisson events in $(0, t-s)$ and is independent of

$$
W_{\alpha, n}(s ; t):=\sum_{j=1}^{N_{n}(s)}\left[\bar{h}_{0}\left(s-T_{j}\right)-\bar{h}_{0}\left(t-T_{j}\right)\right] / \sqrt{n}
$$

thanks to the lack of memory property of the Poisson process. Thus we get, by putting $\rho:=t-s$,

$$
\begin{aligned}
& \mathbb{E}\left[U_{\alpha, n}(t)-U_{\alpha, n}(s)\right]^{4} \\
= & \mathbb{E}\left[U_{\alpha, n}^{*}(\rho)\right]^{4}+\mathbb{E}\left[W_{\alpha, n}(s ; \rho)\right]^{4}-4 \mathbb{E}\left[U_{\alpha, n}^{*}(\rho)\right] \mathbb{E}\left[W_{\alpha, n}(s ; \rho)\right]^{3}+ \\
& -4 \mathbb{E}\left[U_{\alpha, n}^{*}(\rho)\right]^{3} \mathbb{E}\left[W_{\alpha, n}(s ; \rho)\right]+6 \mathbb{E}\left[U_{\alpha, n}^{*}(\rho)\right]^{2} \mathbb{E}\left[W_{\alpha, n}(s ; \rho)\right]^{2} \\
= & \mathbb{E}\left[U_{\alpha, n}^{*}(\rho)\right]^{4}+\mathbb{E}\left[W_{\alpha, n}(s ; \rho)\right]^{4}+6 \mathbb{E}\left[U_{\alpha, n}^{*}(\rho)\right]^{2} \mathbb{E}\left[W_{\alpha, n}(s ; \rho)\right]^{2}
\end{aligned}
$$

since, by the centering, the first moment of both $U_{\alpha, n}^{*}$ and $W_{\alpha, n}(s ; \rho)$ is null. We can now evaluate the moments of $W_{\alpha, n}(s ; \rho)$, by its cumulant generating function:

$$
\begin{aligned}
K(\theta) & :=\log \left\{\mathbb{E} e^{\theta W_{\alpha, n}(s ; \rho)}\right\} \\
& =\log \left\{e^{-\lambda s} \sum_{m=0}^{\infty} \frac{(\lambda s)^{m}}{m !} \mathbb{E}\left[e^{\theta W_{\alpha, n}(s ; \rho)} \mid N(s)=m\right]\right\} \\
& =\log \left\{e^{-\lambda s} \sum_{m=0}^{\infty} \frac{(\lambda s)^{m}}{m !}\left[\frac{1}{s} \int_{0}^{s} e^{\frac{\theta}{\sqrt{n}}\left[\bar{h}_{0}(s-y)-\bar{h}_{0}(s+\rho-y)\right]} d y\right]^{m}\right\} \\
& =\lambda \int_{0}^{s}\left[e^{\frac{\theta}{\sqrt{n}}\left[\bar{h}_{0}(s-y)-\bar{h}_{0}(s+\rho-y)\right]}-1\right] d y,
\end{aligned}
$$

where, in the second line, we have considered the uniform conditional distribution of the occurring time for each Poisson event and their independence. Then, by denoting as $\kappa_{j}$ the $j$-th cumulant of $W_{\alpha, n}(s ; \rho)$ and differentiating $(2.9)$, we get

$\mathbb{E}\left[W_{\alpha, n}(s ; \rho)\right]^{2}=\kappa_{2}-\kappa_{1}^{2}=\left.\frac{d^{2}}{d \theta^{2}} K(\theta)\right|_{\theta=0}=\frac{\lambda}{\sqrt{n}} \int_{0}^{s}\left[\bar{h}_{0}(s-y)-\bar{h}_{0}(s+\rho-y)\right]^{2} d y$

and

$$
\begin{aligned}
\mathbb{E} & {\left[W_{\alpha, n}(s ; \rho)\right]^{4} } \\
= & \kappa_{4}+4 \kappa_{3} \kappa_{1}+3 \kappa_{2}^{2}+6 \kappa_{2} \kappa_{1}^{2}+\kappa_{1}^{4}=\left.\frac{d^{4}}{d \theta^{4}} K(\theta)\right|_{\theta=0}+3\left[\left.\frac{d^{2}}{d \theta^{2}} K(\theta)\right|_{\theta=0}\right]^{2} \\
= & \frac{\lambda}{n^{2}} \int_{0}^{s}\left[\bar{h}_{0}(s-y)-\bar{h}_{0}(s+\rho-y)\right]^{4} d y \\
& +\frac{3 \lambda^{2}}{n}\left\{\int_{0}^{s}\left[\bar{h}_{0}(s-y)-\bar{h}_{0}(s+\rho-y)\right]^{2} d y\right\}^{2}
\end{aligned}
$$

(by considering that the first cumulant vanishes). We now take the sup of (2.8), for $\rho \leq \delta$ :

$$
\begin{aligned}
& \sup _{\rho \leq \delta} \mathbb{E}\left[U_{\alpha, n}(s+\rho)-U_{\alpha, n}(s)\right]^{4} \\
& \leq \sup _{\rho \leq \delta} \mathbb{E}\left[U_{\alpha, n}^{*}(\rho)\right]^{4}+\sup _{\rho \leq \delta} \mathbb{E}\left[W_{\alpha, n}(s ; \rho)\right]^{4}+6 \sup _{\rho \leq \delta} \mathbb{E}\left[U_{\alpha, n}^{*}(\rho)\right]^{2} \sup _{\rho \leq \delta} \mathbb{E}\left[W_{\alpha, n}(s ; \rho)\right]^{2} .
\end{aligned}
$$


All the terms are positive and increasing in $\rho$, for fixed $s$, as can be checked in (2.10) and (2.11), by considering also that

$$
U_{\alpha, n}^{*}(\rho)=\frac{1}{\sqrt{n}} \sum_{j=N_{n}(s)+1}^{N_{n}(s+\rho)} \bar{h}_{0}\left(s+\rho-T_{j}\right) \leq \frac{1}{\sqrt{n}} \sum_{j=N_{n}(s)+1}^{N_{n}(s+\rho)} \bar{h}_{0}\left(s-T_{j}\right)
$$

and that $\bar{h}_{0}(\cdot)$ is positive. Thus we get that

$$
\begin{aligned}
& \sup _{\rho \leq \delta} \mathbb{E}\left[U_{\alpha, n}(s+\rho)-U_{\alpha, n}(s)\right]^{4} \\
& \leq \mathbb{E}\left[U_{\alpha, n}^{*}(\delta)\right]^{4}+\mathbb{E}\left[W_{\alpha, n}(s ; \delta)\right]^{4}+6 \mathbb{E}\left[U_{\alpha, n}^{*}(\delta)\right]^{2} \mathbb{E}\left[W_{\alpha, n}(s ; \delta)\right]^{2} .
\end{aligned}
$$

In order to study the limit for $s \rightarrow \infty$, of (2.12), we note that, for $x, \rho>0$,

$$
\left|\bar{h}_{0}(x)-\bar{h}_{0}(x+\rho)\right| \leq \rho \sup _{x<z<x+\rho} \frac{d}{d z} \bar{h}_{0}(z) \leq \frac{k \rho}{\left(x+\xi_{0}\right)^{\beta}},
$$

for some $k>0$ and $\beta>\frac{3}{2}$. Indeed, by ignoring the expected value, we have that

$$
\begin{aligned}
\frac{d}{d x} & \bar{h}_{0}(x)=\frac{d}{d x}\left[\sqrt{\left(x+\xi_{0}\right)^{\alpha-1} E_{\alpha, \alpha}\left(-\gamma\left(2 x+\xi_{0}\right)^{\alpha}\right)}\right] \\
& =\frac{\sum_{j=0}^{\infty} \frac{\left(-\gamma 2^{\alpha}\right)^{j}\left(x+\xi_{0}\right)^{\alpha j+\alpha-2}}{\Gamma(\alpha j+\alpha-1)}}{2 \sqrt{\left(x+\xi_{0}\right)^{\alpha-1} E_{\alpha, \alpha}\left(-\gamma 2^{\alpha}\left(x+\xi_{0}\right)^{\alpha}\right)}}=\frac{x^{\frac{\alpha}{2}-\frac{3}{2}} E_{\alpha, \alpha-1}\left(-\gamma 2^{\alpha}\left(x+\xi_{0}\right)^{\alpha}\right)}{2 \sqrt{E_{\alpha, \alpha}\left(-\gamma 2^{\alpha}\left(x+\xi_{0}\right)^{\alpha}\right)}} .
\end{aligned}
$$

Whence, using (1.9),

$$
\begin{aligned}
\sup _{x<z<x+\rho} \frac{d}{d z} \bar{h}_{0}(z) & \leq \sup _{z \geq x} \frac{d}{d z} \bar{h}_{0}(z) \\
& \leq \sup _{z \geq x} \frac{\left[K_{1}\left(z+\xi_{0}\right)^{-2 \alpha}+O\left(z^{-3 \alpha}\right)\right] / \Gamma(-\alpha-1)}{\left(z+\xi_{0}\right)^{\frac{3}{2}-\frac{\alpha}{2}}\left[K_{2}\left(z+\xi_{0}\right)^{-2 \alpha}+O\left(z^{-3 \alpha}\right)\right]^{1 / 2} / \Gamma(-\alpha)} \\
& \leq \frac{K_{3}}{\left(z+\xi_{0}\right)^{\beta}}
\end{aligned}
$$

(for some positive constants $K_{1}, K_{2} K_{3}$, so that (2.13) is satisfied with $\beta=\frac{\alpha}{2}+\frac{3}{2}$ ). As a consequence,

$$
\begin{aligned}
& \int_{0}^{s}\left[\bar{h}_{0}(s-y)-\bar{h}_{0}(s+\rho-y)\right]^{2} d y \\
= & \int_{0}^{s-c}\left[\bar{h}_{0}(s-y)-\bar{h}_{0}(s+\rho-y)\right]^{2} d y+\int_{s-c}^{s}\left[\bar{h}_{0}(s-y)-\bar{h}_{0}(s+\rho-y)\right]^{2} d y .
\end{aligned}
$$

The second integral can be bounded by considering that, for any $c>0$ and $u \in[0, c]$,

$$
\begin{aligned}
\left|\bar{h}_{0}(u)-\bar{h}_{0}(u+\rho)\right| & \leq \rho \sup _{0 \leq z \leq c} \frac{d}{d z} \bar{h}_{0}(z) \\
& =[\text { analogously to }(2.14)] \\
& \leq \frac{K_{3} \rho}{\xi_{0}^{\beta}} .
\end{aligned}
$$


The first moments of the limiting process $U_{\alpha}$ can be evaluated by differentiating (2.6) and reads: $\mathbb{E} U_{\alpha}(t)=0$ and

$$
\begin{aligned}
& \operatorname{Var}\left(U_{\alpha}(t)\right)=\lambda_{0} \int_{-\infty}^{+\infty} h_{0}^{2}(t-u) d u \\
& =\lambda_{0} \int_{0}^{t}\left(u+\xi_{0}\right)^{\alpha-1} E_{\alpha, \alpha}\left(-\gamma 2^{\alpha}\left(u+\xi_{0}\right)^{\alpha}\right) d u \\
& =-\left.\lambda_{0} E_{\alpha, 1}\left(-\gamma 2^{\alpha}\left(u+\xi_{0}\right)^{\alpha}\right)\right|_{u=0} ^{t}=\lambda_{0}\left[E_{\alpha, 1}\left(-\gamma 2^{\alpha} \xi_{0}^{\alpha}\right)-E_{\alpha, 1}\left(-\gamma 2^{\alpha}\left(t+\xi_{0}\right)^{\alpha}\right)\right]
\end{aligned}
$$

by considering that

$$
\frac{d}{d w} E_{\alpha, 1}\left(-k w^{\alpha}\right)=-k w^{\alpha-1} E_{\alpha, \alpha}\left(-k w^{\alpha}\right), \quad k, w \in \mathbb{R} .
$$

The previous Lemma shows that the distribution of a suitably normalized sequence of Poisson shot-noise (with power-law impulse response function described by $h_{0}$ ) converges, when the frequency of shocks is large and their amplitude small, to a generalized version of the OU process. Indeed, for $\xi_{0}=0$, one obtains the following one-dimensional distribution

$$
\begin{aligned}
f_{U_{\alpha}(t)}(x) & :=P\left\{U_{\alpha}(t) \in d x\right\} / d x \\
& =\frac{1}{\sqrt{2 \pi(\gamma / \theta)\left[1-E_{\alpha, 1}\left(-\gamma(2 t)^{\alpha}\right)\right]}} \exp \left\{-\frac{x^{2}}{2(\gamma / \theta)\left[1-E_{\alpha, 1}\left(-\gamma(2 t)^{\alpha}\right)\right]}\right\},
\end{aligned}
$$

for $x \in \mathbb{R}, t>0, \gamma, \theta>0$. Note that, as far as the convergence of the finitedimensional distributions is concerned, $\xi_{0}$ could be allowed to take any fixed nonnegative value $\xi_{0} \in \mathbb{R}_{0}^{+}$; however the proof of tightness requires the slightly stronger condition $\xi_{0}>0$.

\section{Main results}

3.1. Time-changed Ornstein-Uhlenbeck process. We start by evaluating the solution of a fractional version of the Fokker-Planck equation (in the Fourier space), defined by means of the operator introduced above.

Theorem 3.1. Let $\mathcal{L}_{t}^{\alpha}$ be the operator defined in Def.1.1, then the solution of the following equation

$\mathcal{L}_{t}^{\alpha} \widehat{u}(\xi, t)=-\frac{\gamma}{2^{1-\alpha}} \xi \frac{\partial}{\partial \xi} \widehat{u}(\xi, t)-\frac{\theta}{2^{1-\alpha}} \xi^{2} \widehat{u}(\xi, t), \quad \xi \in \mathbb{R}, t \geq 0, \alpha \in(0,1], D, \gamma>0$,

with initial condition $\widehat{u}(\xi, 0)=1$, is given by

$$
\widehat{u}(\xi, t)=\exp \left\{-\frac{\theta \xi^{2}}{2 \gamma}\left[1-E_{\alpha, 1}\left(-\gamma(2 t)^{\alpha}\right)\right]\right\} .
$$

Proof: We start by writing the Fourier transform of the FP equation, given in (1.1), i.e.

$$
\begin{aligned}
& \frac{\partial}{\partial t} \widehat{u}(\xi, t)=\eta \int_{-\infty}^{+\infty} e^{i \xi x} \frac{\partial}{\partial x}[x u(x, t)] d x-D \xi^{2} \widehat{u}(\xi, t) \\
= & \eta\left\{\left[e^{i \xi x} x u(x, t)\right]_{-\infty}^{+\infty}-i \xi \int_{-\infty}^{+\infty} e^{i \xi x} x u(x, t) d x\right\}-D \xi^{2} \widehat{u}(\xi, t),
\end{aligned}
$$


which, with $\eta=\gamma / 2^{1-\alpha}$ and $D=\theta / 2^{1-\alpha}$, coincides with the r.h.s. of (3.1), by considering the boundary condition.

Since the Mittag-Leffler function (1.7) is the eigenfunction of the Caputo fractional derivative (1.2) (see Lemma 2.23, p.98, in Kilbas et al., 2006), we get from (3.2), that

$$
\begin{aligned}
D_{t}^{\alpha} \log \widehat{u}(\xi, t) & =-\frac{\theta \xi^{2}}{2 \gamma} D_{t}^{\alpha}\left[1-E_{\alpha, 1}\left(-\gamma(2 t)^{\alpha}\right)\right] \\
& =-\frac{\theta \xi^{2}}{2^{1-\alpha}} E_{\alpha, 1}\left(-\gamma(2 t)^{\alpha}\right) \\
& =-2^{\alpha} \gamma \log \widehat{u}(\xi, t)-\frac{\theta \xi^{2}}{2^{1-\alpha}}
\end{aligned}
$$

and thus

$$
\widehat{u}(\xi, t) D_{t}^{\alpha} \log \widehat{u}(\xi, t)=\frac{\theta \xi^{2}}{2^{1-\alpha}}\left[1-E_{\alpha, 1}\left(-\gamma(2 t)^{\alpha}\right)\right] \widehat{u}(\xi, t)-\frac{\theta \xi^{2}}{2^{1-\alpha}} \widehat{u}(\xi, t),
$$

which coincides with (3.1). The initial condition is verified since $E_{\alpha, 1}(0)=1$.

Remark 3.2. Formula (3.2) coincides with the characteristic function of the process $U_{\alpha}(t)$ obtained, in the previous section, by taking the limit of the power-law Poisson shot-noise defined in Def.2.2. Moreover, for $\alpha=1$, it reduces to the characteristic function of the OU process (with starting point in the origin), i.e.

$$
\widehat{u}(\xi, t)=\exp \left\{-\frac{\theta \xi^{2}}{2 \gamma}\left[1-e^{-2 \gamma t}\right]\right\} .
$$

Similarly to the result obtained in Lemma 2.1, the solution of (3.1) coincides with the characteristic function of the OU process time-changed by means of the continuous and increasing function

$$
\mathcal{T}_{\alpha}(t)=-\frac{1}{2 \gamma} \log E_{\alpha, 1}\left(-\gamma(2 t)^{\alpha}\right)
$$

such that $\mathcal{T}_{\alpha}(0)=0$. Therefore we define the time-changed OU process, by means of (3.3), as follows.

Definition 3.3. (Time-changed OU process) Let $X_{1}:=\left\{X_{1}(t), t \geq 0\right\}$ denote the standard OU process, then we define the time-changed OU process $X_{\alpha}=$ $\left\{X_{\alpha}(t), t \geq 0\right\}$, as $X_{\alpha}(t):=X_{1}\left(\mathcal{T}_{\alpha}(t)\right)$, for $\mathcal{T}_{\alpha}(\cdot)$ given in (3.3).

Remark 3.4. The process $X_{\alpha}$ is still Gaussian (being obtained by a deterministic time-change) with $\mathbb{E} X_{\alpha}(t)=0$, and, for any $t, s \geq 0, \gamma>0, \alpha \in(0,1]$, has

$$
\operatorname{Cov}\left(X_{\alpha}(t), X_{\alpha}(s)\right)=\frac{\theta}{\gamma} \sqrt{\frac{E_{\alpha, 1}\left(-\gamma 2^{\alpha}(t \vee s)^{\alpha}\right)}{E_{\alpha, 1}\left(-\gamma 2^{\alpha}(t \wedge s)^{\alpha}\right)}}\left[1-E_{\alpha, 1}\left(-\gamma 2^{\alpha}(t \wedge s)^{\alpha}\right)\right] .
$$

Formula (3.4) can be derived as follows

$$
\begin{aligned}
& \operatorname{Cov}\left(X_{\alpha}(t), X_{\alpha}(s)\right)=\frac{\theta}{\gamma} e^{-\gamma \mathcal{T}_{\alpha}(t) \mid}\left[e^{\gamma \mathcal{T}_{\alpha}(s)}-e^{-\gamma \mathcal{T}_{\alpha}(s)}\right] \\
& =\frac{\theta}{\gamma} e^{\frac{1}{2} \log E_{\alpha, 1}\left(-\gamma(2 t)^{\alpha}\right)}\left[e^{-\frac{1}{2} \log E_{\alpha, 1}\left(-\gamma(2 s)^{\alpha}\right)}-e^{\frac{1}{2} \log E_{\alpha, 1}\left(-\gamma(2 s)^{\alpha}\right)}\right]
\end{aligned}
$$

for $s<t$ and thus for $\mathcal{T}_{\alpha}(s)<\mathcal{T}_{\alpha}(t)$, by recalling that the Mittag-Leffler function $E_{\alpha, 1}(x)$ is completely monotone, for $\alpha \in(0,1)$, on the real negative semi-axis. Analogously, for $s \geq t$. 
Remark 3.5. We can easily derive the following representation of $X_{\alpha}$ in terms of a Brownian motion, by applying again the time change (3.3) to the well known representation of the standard OU process:

$$
\begin{aligned}
X_{\alpha}(t) & =\sqrt{\frac{\theta}{\gamma}} e^{-\gamma \mathcal{T}_{\alpha}(t)} W\left(e^{2 \gamma \mathcal{T}_{\alpha}(t)}-1\right) \\
& =\sqrt{\frac{\theta}{\gamma} E_{\alpha, 1}\left(-2^{\alpha} \gamma t^{\alpha}\right)} W\left(\frac{1}{E_{\alpha, 1}\left(-2^{\alpha} \gamma t^{\alpha}\right)}-1\right) .
\end{aligned}
$$

Moreover, it follows from (3.4) that the process is Markovian, for any $\alpha$, since

$$
\rho_{X_{\alpha}}(s, t)=\sqrt{\frac{E_{\alpha, 1}\left(-\gamma 2^{\alpha} t^{\alpha}\right)\left[1-E_{\alpha, 1}\left(-\gamma 2^{\alpha} s^{\alpha}\right)\right]}{E_{\alpha, 1}\left(-\gamma 2^{\alpha} s^{\alpha}\right)\left[1-E_{\alpha, 1}\left(-\gamma 2^{\alpha} t^{\alpha}\right)\right]}}=\rho_{X_{\alpha}}(s, h) \rho_{X_{\alpha}}(h, t), \quad s<h<t,
$$

where $\rho_{X_{\alpha}}(\cdot, \cdot)$ denotes the autocorrelation coefficient. On the other hand, in this case, by considering formula (1.9), we get, for any $\tau \geq 0$,

$$
\begin{aligned}
& \lim _{t \rightarrow \infty} \operatorname{Cov}\left(X_{\alpha}(t), X_{\alpha}(t+\tau)\right) \\
& =\frac{\theta}{\gamma} \lim _{t \rightarrow \infty} \sqrt{\frac{E_{\alpha, 1}\left(-\gamma 2^{\alpha}(t+\tau)^{\alpha}\right)}{E_{\alpha, 1}\left(-\gamma 2^{\alpha} t^{\alpha}\right)}}\left[1-E_{\alpha, 1}\left(-\gamma 2^{\alpha} t^{\alpha}\right)\right]=\frac{\theta}{\gamma} .
\end{aligned}
$$

Thus the process $X_{\alpha}$ asymptotically behaves as a random walk. Moreover it does not coincide, in the limit, with the stationary OU process (which we denote by $\bar{X}_{1}$ ) time-changed by (3.3), i.e. with $\bar{X}_{\alpha}(t)=\bar{X}_{1}\left(\mathcal{T}_{\alpha}(t)\right)$, for $\mathcal{T}_{\alpha}(\cdot)$ given in (3.3). Indeed the latter is a Gaussian (non-stationary) process with $\mathbb{E} \bar{X}_{\alpha}(t)=0$, and

$$
\operatorname{Cov}\left(\bar{X}_{\alpha}(t), \bar{X}_{\alpha}(s)\right)=\frac{\theta}{\gamma} \sqrt{\frac{E_{\alpha, 1}\left(-\gamma 2^{\alpha}(t \vee s)^{\alpha}\right)}{E_{\alpha, 1}\left(-\gamma 2^{\alpha}(t \wedge s)^{\alpha}\right)}},
$$

for any $t, s \geq 0, \gamma>0, \alpha \in(0,1]$. Formula (3.6) is obtained as follows: for $t>s$

$$
\begin{aligned}
\operatorname{Cov}\left(\bar{X}_{\alpha}(t), \bar{X}_{\alpha}(s)\right) & =\frac{\theta}{\gamma} e^{-\gamma\left|\mathcal{T}_{\alpha}(t)-\mathcal{T}_{\alpha}(s)\right|}=\frac{\theta}{\gamma} e^{\frac{1}{2}\left[\log E_{\alpha, 1}\left(-\gamma 2^{\alpha} t^{\alpha}\right)-\log E_{\alpha, 1}\left(-\gamma 2^{\alpha} s^{\alpha}\right)\right]} \\
& =\frac{\theta}{\gamma} \sqrt{\frac{E_{\alpha, 1}\left(-\gamma 2^{\alpha} t^{\alpha}\right)}{E_{\alpha, 1}\left(-\gamma 2^{\alpha} s^{\alpha}\right)}}
\end{aligned}
$$

3.2. Fractional OU process. In order to overcome the anomalous asymptotic behavior of the previous model, we start by defining the fractional Ornstein-Uhlenbeck process in the stationary case, by means of the following preliminary result.

Lemma 3.6. The function

$$
f(s)=E_{\alpha, 1}\left(-\gamma|s|^{\alpha}\right), s \in \mathbb{R},
$$

is positive definite.

Proof: We apply the Schoenberg's characterization: let $\varphi:[0,+\infty) \rightarrow \mathbb{R}$ be a continuous function, then $\Phi(\cdot)=\varphi\left(\|\cdot\|^{2}\right)$ is positive definite and radial on $\mathbb{R}^{d}$, for any $d$, if and only if $\varphi(\cdot)$ is $\mathrm{CM}$ on $[0,+\infty)$. Then, we note that the complete monotonicity of the Mittag-Leffler function of negative argument and that the composition of a CM functional and a Bernstein function is again CM (see Mainardi, 2014 for 
details). Since $s^{\alpha / 2}$ is a Bernstein function, the result follows by considering that $E_{\alpha, 1}\left(-\gamma s^{\alpha / 2}\right)$ is CM.

With this at hand we can give the following definition:

Definition 3.7. (Fractional stationary OU) Let $\overline{\mathcal{Y}}_{\alpha}:=\left\{\overline{\mathcal{Y}}_{\alpha}(t), t \geq 0\right\}$ be defined as a Gaussian process with $\mathbb{E} \overline{\mathcal{Y}}_{\alpha}(t)=0$, for any $t \geq 0$ and

$$
r(s):=\operatorname{Cov}\left(\overline{\mathcal{Y}}_{\alpha}(t), \overline{\mathcal{Y}}_{\alpha}(t+s)\right)=\frac{\theta}{\gamma} E_{\alpha, 1}\left(-\gamma|s|^{\alpha}\right),
$$

for any $s \in \mathbb{R}, \gamma>0, \alpha \in(0,1]$.

Remark 3.8. Long-range dependent processes with autocorrelation function decaying as a Mittag-Leffler have been already studied in Barndorff-Nielsen and Leonenko (2005), where they are obtained, in a completely different way, i.e. by superposition of OU processes.

Then, for the spectral density of the fractional stationary OU defined above, we prove the following result.

Lemma 3.9. The spectral density of the process $\overline{\mathcal{Y}}_{\alpha}$ is given by

$$
\mathcal{S}_{\overline{\mathcal{Y}}_{\alpha}}(\omega)=\frac{\theta}{\sqrt{\pi} \gamma} H_{2,3}^{2,1}\left[\frac{|\omega|^{\alpha}}{2^{\alpha} \gamma} \mid \begin{array}{ccc}
(0,1) & (1-\alpha, \alpha) & \left(\frac{1}{2}-\frac{\alpha}{2}, \frac{\alpha}{2}\right) \\
(0,1) & \left(1-\frac{\alpha}{2}, \frac{\alpha}{2}\right)
\end{array}\right], \quad \omega \in \mathbb{R} \backslash\{0\},
$$

where $H_{p, q}^{m, n}$ is the H-function defined in (1.10). Then its spectral representation reads

$$
\overline{\mathcal{Y}}_{\alpha}(t)=\int_{-\infty}^{+\infty} \sqrt{\mathcal{S}_{\overline{\mathcal{Y}}_{\alpha}}(\omega)} e^{i t \omega} d W(\omega)
$$

Proof: From (3.7) we can write, for any $t \geq 0$ and $\omega \in \mathbb{R}$,

$$
\begin{aligned}
\mathcal{S}_{\overline{\mathcal{Y}}_{\alpha}}(\omega) & =\frac{1}{2 \pi} \int_{-\infty}^{+\infty} e^{-i \omega s} \operatorname{Cov}\left(\overline{\mathcal{Y}}_{\alpha}(t), \overline{\mathcal{Y}}_{\alpha}(t+s)\right) d s \\
& =\frac{\theta}{\pi \gamma} \int_{0}^{+\infty} \cos (|\omega| s) E_{\alpha, 1}\left(-\gamma s^{\alpha}\right) d s \\
& =[\text { by }(1.12 .65) \text { in Kilbas et al. }(2006)] \\
& =\frac{\theta}{\pi \gamma} \int_{0}^{+\infty} \cos (|\omega| s) H_{1,2}^{1,1}\left[\gamma s^{\alpha} \mid \begin{array}{c}
(0,1) \\
(0,1)(0, \alpha)
\end{array}\right] d s .
\end{aligned}
$$

In order to evaluate the previous cosine transform, we apply formula (2.50), for $\rho=1$, of Mathai et al. (2010), i.e.

$$
\begin{aligned}
& \int_{0}^{+\infty} \cos (a x) H_{p, q}^{m, n}\left[b x^{\sigma} \mid \begin{array}{c}
\left(a_{p}, A_{p}\right) \\
\left(b_{q}, B_{q}\right)
\end{array}\right] d t \\
& =\frac{\sqrt{\pi}}{a} H_{p+2, q}^{m, n+1}\left[b\left(\frac{2}{a}\right)^{\sigma} \mid \begin{array}{ccc}
\left(\frac{1}{2}, \frac{\sigma}{2}\right) \\
\left(b_{q}, B_{q}\right)
\end{array}\right.
\end{aligned}
$$

for $a, \alpha, \sigma>0, b \in \mathbb{C}, \mathcal{R}\left[1+\sigma \max _{1 \leq j \leq n}\left\{\frac{a_{j}-1}{A_{j}}\right\}\right]<1$ and $\mathcal{R}\left[1+\sigma \min _{1 \leq j \leq m}\left\{\frac{b_{j}}{B_{j}}\right\}\right]$ $>0 ; \arg |a|<\pi \alpha / 2$, where $\alpha=\sum_{j=1}^{m} B_{j}-\sum_{j=m+1}^{q} B_{j}+\sum_{j=1}^{n} A_{j}-\sum_{j=n+1}^{p} A_{j}$. 
It is easy to check that the corresponding conditions are all satisfied, since $1+$ $\alpha\left\{\frac{a_{1}-1}{A_{1}}\right\}=1-\alpha<1,1+\alpha \frac{b_{1}}{B_{1}}=1>0$ and $\alpha=B_{1}-B_{2}+A_{1}=2-\alpha>0$. Then (3.10) becomes

$$
\begin{aligned}
& \mathcal{S}_{\overline{\mathcal{Y}}_{\alpha}}(\omega)=\frac{\theta}{\sqrt{\pi} \gamma|\omega|} H_{3,2}^{1,2}\left[\frac{2^{\alpha} \gamma}{|\omega|^{\alpha}} \mid \begin{array}{ccc}
\left(\frac{1}{2}, \frac{\alpha}{2}\right) & (0,1) & \left(0, \frac{\alpha}{2}\right) \\
(0,1) & (0, \alpha)
\end{array}\right] \\
& =[\text { by (1.12.47) in Kilbas et al. (2006)] } \\
& =\frac{\theta}{\sqrt{\pi} \gamma|\omega|} H_{2,3}^{2,1}\left[\frac{|\omega|^{\alpha}}{2^{\alpha} \gamma} \mid \begin{array}{ccc}
(1,1) & (1, \alpha) & \\
\left(\frac{1}{2}, \frac{\alpha}{2}\right) & (1,1) & \left(1, \frac{\alpha}{2}\right)
\end{array}\right],
\end{aligned}
$$

which coincides with (3.8), by applying formula (1.12..45) in Kilbas et al. (2006), for $\sigma=-1 / \alpha$. The existence condition in Mathai et al. (2010) is satisfied, for any $\omega \neq 0$, by (3.8), since $\alpha=\alpha / 2>0$, by choosing the contour $\mathcal{L}=\mathcal{L}_{i \gamma \infty}$, since in this case $\mu=\sum_{j=1}^{q} B_{j}-\sum_{j=1}^{p} A_{j}=0$ (see Theorem 1.1, case 7, in Mathai et al. (2010)). Finally, the function (3.8) is positive, since (3.7) is positive definite, by the Bochner's theorem. The representation (3.9) easily follows.

Remark 3.10. We can check that in the special case $\alpha=1$ formula (3.8) reduces to the well-known spectral density of the OU process:

$$
\begin{aligned}
& \mathcal{S}_{\overline{\mathcal{Y}}_{\alpha}}(\omega)=\frac{\theta}{2 \gamma \sqrt{\pi}} H_{2,3}^{2,1}\left[\frac{|\omega|}{2 \gamma} \mid \begin{array}{lll}
(0,1) & (0,1) & \\
\left(0, \frac{1}{2}\right) & (0,1) & \left(\frac{1}{2}, \frac{1}{2}\right)
\end{array}\right] \\
& =\frac{\theta}{2 \gamma \sqrt{\pi}} \frac{1}{2 \pi i} \int_{\mathcal{L}}\left(\frac{|\omega|}{2 \gamma}\right)^{-s} \frac{\Gamma\left(\frac{s}{2}\right) \Gamma(1-s)}{\Gamma\left(\frac{1}{2}-\frac{s}{2}\right)} d s \\
& =\text { [by the duplication formula] } \\
& =\frac{\theta}{2 \pi \gamma} \frac{1}{2 \pi i} \int_{\mathcal{L}}\left(\frac{|\omega|}{\gamma}\right)^{-s} \Gamma\left(\frac{s}{2}\right) \Gamma\left(1-\frac{s}{2}\right) d s \\
& =\frac{\theta}{2 \pi \gamma} H_{1,1}^{1,1}\left[\frac{|\omega|}{\gamma} \mid \begin{array}{l}
\left(0, \frac{1}{2}\right) \\
\left(0, \frac{1}{2}\right)
\end{array}\right] \\
& =\text { see (4.8) in Vellaisamy and Kataria (2018)] } \\
& =\frac{\theta \gamma}{\pi} \frac{1}{\gamma^{2}+\omega^{2}} \text {. }
\end{aligned}
$$

for $\omega \in \mathbb{R}$.

In order to derive the dependence properties of the process $\overline{\mathcal{Y}}_{\alpha}$ we apply Theorem 1.2, p.19, in Mathai et al. (2010): the asymptotic behavior, for $|\omega| \rightarrow 0$, of the spectral density (3.11) is then given by

$$
\mathcal{S}_{\overline{\mathcal{Y}}_{\alpha}}(\omega)=\frac{1}{|\omega|} O\left(|\omega|^{\alpha}\right)=O\left(|\omega|^{\alpha-1}\right), \quad|\omega| \rightarrow 0,
$$

since $c=\min [1 / \alpha, 1]=1$. As a consequence, we can conclude that the process $\overline{\mathcal{Y}}_{\alpha}$ exhibits long-range dependence under the assumption that $\alpha \in(0,1)$, while for $\alpha=1$ we have $\frac{\mathcal{S}_{\mathcal{y}}(\omega)}{|\omega|^{a}} \rightarrow \infty$, for any $a \in(0,1)$, as it is well-known in the standard OU case. The same conclusion could be drawn form the covariance function (3.7), taking into account (1.9), so that $r(s) \sim C_{\alpha} s^{-\alpha}$, for some constant $C_{\alpha}$ and $s \rightarrow+\infty$. Thus the process is long-memory of order $1-\alpha$, both in the covariance and in the spectral density sense. 
We prove the following properties for the trajectories of the fractional stationary OU process.

Theorem 3.11. There exists a version of $\overline{\mathcal{Y}}_{\alpha}$ with continuous trajectories and the latter are not differentiable in the $L^{2}$-norm.

Proof: In order to apply the Kolmogorov continuity theorem, we only need to check that there exist $p, \eta>1$, such that, for a constant $c$ and for any $t_{1}, t_{2} \in I$, the following inequality holds:

$$
\mathbb{E}\left|\overline{\mathcal{Y}}_{\alpha}\left(t_{2}\right)-\overline{\mathcal{Y}}_{\alpha}\left(t_{1}\right)\right|^{p} \leq c\left|t_{2}-t_{1}\right|^{\eta} .
$$

Let $\lceil x\rceil$ denote the upper integer part of $x \in \mathbb{R}$, then, by choosing $p=\lceil 2 / \alpha\rceil$, $\eta=\frac{\alpha p}{2}>1$, the previous inequality is verified; indeed

$$
\begin{aligned}
\mathbb{E}\left|\overline{\mathcal{Y}}_{\alpha}\left(t_{2}\right)-\overline{\mathcal{Y}}_{\alpha}\left(t_{1}\right)\right|^{p} & =\frac{2^{p} \Gamma((p+1) / 2)}{\sqrt{\pi} \gamma^{p / 2}}\left[\mathbb{E}\left[\overline{\mathcal{Y}}_{\alpha}\left(t_{2}\right)-\overline{\mathcal{Y}}_{\alpha}\left(t_{1}\right)\right]^{2}\right]^{p / 2} \\
& =C_{p}\left[1-E_{\alpha, 1}\left(-\gamma\left|t_{2}-t_{1}\right|^{\alpha}\right)\right]^{p / 2} \\
& =\gamma^{p / 2} C_{p}\left|t_{2}-t_{1}\right|^{\alpha / 2}\left[E_{\alpha, \alpha+1}\left(-\gamma\left|t_{2}-t_{1}\right|^{\alpha}\right)\right]^{p / 2} \\
& \leq \frac{\gamma^{p / 2} C_{p}}{\Gamma(\alpha+1)^{p / 2}}\left|t_{2}-t_{1}\right|^{\alpha p / 2},
\end{aligned}
$$

(where $\left.C_{p}=2^{p} \theta^{p / 2} \Gamma((p+1) / 2) / \gamma^{p / 2} \sqrt{\pi}\right)$, since $E_{\alpha, \alpha+1}\left(-\gamma\left|t_{2}-t_{1}\right|^{\alpha}\right) \leq 1 / \Gamma(\alpha+1)$. Indeed $E_{\alpha, \alpha+1}(0)=1 / \Gamma(\alpha+1)$ and the function is completely monotone and nonincreasing (see Gorenflo et al., 2014, Sec. 4.10.2). Moreover we have that

$$
\mathbb{E}\left(\frac{\overline{\mathcal{Y}}_{\alpha}\left(t_{2}\right)-\overline{\mathcal{Y}}_{\alpha}\left(t_{1}\right)}{t_{2}-t_{1}}\right)^{2}=2 \theta\left|t_{2}-t_{1}\right|^{\alpha-2} E_{\alpha, \alpha+1}\left(-\gamma\left|t_{2}-t_{1}\right|^{\alpha}\right) \rightarrow+\infty,
$$

for $t_{2} \rightarrow t_{1}$ and $\alpha \in(0,1]$, by considering that $\lim _{x \rightarrow 0} E_{\alpha, \alpha+1}\left(-x^{\alpha}\right)=1 / \Gamma(\alpha+1)$. Alternatively, we could notice that the covariance function can be expanded as follows: $r(\tau)=r(0)-\frac{\gamma|\tau|^{\alpha}}{\Gamma(\alpha+1)}+o\left(|\tau|^{\alpha}\right)$.

We can now define a non-stationary version of the fractional OU process, whose density function coincides with the solution of the fractional Fokker-Planck equation (3.1), in Fourier space. We start by the following preliminary result.

Lemma 3.12. The function

$$
f(s)=\sqrt{E_{\alpha, 1}\left(-k s^{\alpha}\right)}, s \in \mathbb{R}, k>0
$$

is positive definite.

Proof: Similarly to Lemma 2.1, the result follows by proving that $\sqrt{E_{\alpha, 1}\left(-\gamma s^{\alpha / 2}\right)}$ is CM. But, as can be checked directly, by differentiating, if $f(t)$ is a CM function, then the same is true for $\sqrt{f(t)}$.

Definition 3.13. (Fractional OU) Let $\left(\Omega, \mathcal{F}, \mathcal{F}_{t}, P\right)$ be a probability space with filtration $\mathcal{F}_{t}$ and let $\mathcal{Y}_{\alpha}:=\left\{\mathcal{Y}_{\alpha}(t), t \geq 0\right\}$ be defined as follows

$$
\mathcal{Y}_{\alpha}(t)=\overline{\mathcal{Y}}_{\alpha}(t)-\sqrt{E_{\alpha, 1}\left(-\gamma(2 t)^{\alpha}\right)} \mathcal{Z}
$$


where $\left(\overline{\mathcal{Y}}_{\alpha}(t), \mathcal{Z}\right)$ is a Gaussian centered random vector with covariance matrix

$$
\sum=\left(\begin{array}{cc}
\frac{\theta}{\gamma} & \frac{\theta}{\gamma} \sqrt{E_{\alpha, 1}\left(-\gamma(2 t)^{\alpha}\right)} \\
\frac{\theta}{\gamma} \sqrt{E_{\alpha, 1}\left(-\gamma(2 t)^{\alpha}\right)} & \frac{\theta}{\gamma}
\end{array}\right),
$$

for any $t \geq 0$.

Remark 3.14. The fractional OU process $\mathcal{Y}_{\alpha}$ is centered, Gaussian with

$$
\operatorname{Cov}\left(\mathcal{Y}_{\alpha}(t), \mathcal{Y}_{\alpha}(s)\right)=\frac{\theta}{\gamma}\left[E_{\alpha, 1}\left(-\gamma|t-s|^{\alpha}\right)-\sqrt{E_{\alpha, 1}\left(-\gamma(2 t)^{\alpha}\right) E_{\alpha, 1}\left(-\gamma(2 s)^{\alpha}\right)}\right],
$$

for $s, t \geq 0, \alpha \in(0,1]$, and $\theta, \gamma>0$. Since $\mathcal{Y}_{\alpha}$ is a zero-mean Gaussian process, its finite-dimensional distributions are completely characterized by its autocovariance function. The variance of the process reads

$$
\operatorname{Var}_{\alpha}(t)=\frac{\theta}{\gamma}\left[1-E_{\alpha, 1}\left(-\gamma(2 t)^{\alpha}\right)\right]
$$

which is positive since $E_{\alpha, 1}\left(-x^{\alpha}\right) \leq 1$, for $x \geq 0$. Thus, the one-dimensional density of the process $\mathcal{Y}_{\alpha}$ coincides with the solution to the fractional FP equation (3.1) and its characteristic function is given in (3.2). By considering (1.9), we notice that the process $\mathcal{Y}_{\alpha}$ is a mean reverting process, for any $\alpha$, since $\lim _{t \rightarrow \infty} \operatorname{Var} \mathcal{Y}_{\alpha}(t)=\theta / \gamma$. Moreover from (3.13) it is evident that the process is non-Markovian for $\alpha \neq 1$, since

$$
\begin{aligned}
\rho_{\mathcal{Y}_{\alpha}}(s, t) & =\frac{E_{\alpha, 1}\left(-\gamma|t-s|^{\alpha}\right)-\sqrt{E_{\alpha, 1}\left(-\gamma(2 t)^{\alpha}\right) E_{\alpha, 1}\left(-\gamma(2 s)^{\alpha}\right)}}{\sqrt{1-E_{\alpha, 1}\left(-\gamma(2 t)^{\alpha}\right)} \sqrt{1-E_{\alpha, 1}\left(-\gamma(2 s)^{\alpha}\right)}} \\
& \neq \rho_{\mathcal{Y}_{\alpha}}(s, h) \rho \mathcal{Y}_{\alpha}(h, t),
\end{aligned}
$$

for $s, h, t \geq 0$. Only for $\alpha=1$, the sufficient condition for the Markov property (in the Gaussian case) is verified: indeed the process $\mathcal{Y}_{1}$ coincides with the standard OU process, since

$$
\operatorname{Cov}\left(\mathcal{Y}_{1}(t), \mathcal{Y}_{1}(s)\right)=\frac{\theta}{\gamma}\left[e^{-\gamma|t-s|}-e^{-\gamma|t+s|}\right]=\operatorname{Cov}(X(t), X(s)) .
$$

Thus, for $\alpha \neq 1$, the process must also have dependent increments, otherwise the Markovian property would follow from Gaussianity.

Theorem 3.15. The cumulant generating function $C_{\mathcal{Y}_{\alpha}}(\eta, t):=\log \mathbb{E} e^{\eta \mathcal{Y}_{\alpha}(t)}$ of $\mathcal{Y}_{\alpha}$, i.e.

$$
C_{\mathcal{Y}_{\alpha}}(\eta, t)=\frac{\eta^{2} \theta}{2 \gamma}\left[1-E_{\alpha, 1}\left(-\gamma(2 t)^{\alpha}\right)\right]
$$

satisfies the following fractional equation

$$
D_{t}^{\alpha} u(\eta, t)=-2^{\alpha} \gamma u(\eta, t)+2^{\alpha-1} \eta^{2} \theta
$$

with $u(\eta, 0)=0$.

Proof: Let $\widetilde{f}(s):=\mathcal{L}\{f(t) ; s\}=\int_{0}^{+\infty} e^{-s t} f(t) d t$ denote the Laplace transform of $f(\cdot)$. By considering the well-known formula (see Kilbas et al., 2006, Lemma 2.24)

$$
\mathcal{L}\left\{D_{t}^{\alpha} f(t) ; s\right\}=s^{\alpha} \widetilde{f}(s)-\left.\sum_{j=0}^{m-1} s^{\alpha-j-1} \frac{d^{n}}{d t^{n}} f(t)\right|_{t=0}, \quad m-1<\alpha \leq m,
$$


the Laplace transform of (3.15) reads

$$
s^{\alpha} \widetilde{u}(\eta, s)-s^{\alpha-1} u(\eta, 0)=-2^{\alpha} \gamma \widetilde{u}(\eta, s)+\frac{2^{\alpha-1} \eta^{2} \theta}{s} .
$$

Then, by the initial condition, we get

$$
\widetilde{u}(\eta, s)=\frac{2^{\alpha-1} \eta^{2} \theta s^{-1}}{s^{\alpha}+2^{\alpha} \gamma},
$$

which, by means of (1.8), gives

$$
u(\eta, t)=2^{\alpha-1} \eta^{2} \theta t^{\alpha} E_{\alpha, \alpha+1}\left(-\gamma(2 t)^{\alpha}\right)=2^{\alpha-1} \eta^{2} \theta t^{\alpha} \sum_{j=0}^{\infty} \frac{\left(-2^{\alpha} \gamma t^{\alpha}\right)^{j}}{\Gamma(\alpha j+\alpha+1)}
$$

which, after some algebra, coincides with (3.14). The latter satisfies the initial conditions since $E_{\alpha, 1}(0)=1$.

Remark 3.16. We notice that a similar result holds for the moment generating function of the time-changed Brownian motion (time-fractional diffusion), i.e. $W\left(\mathcal{L}_{\alpha}(t)\right)$, where $W$ denotes a standard Brownian motion and $\mathcal{L}_{\alpha}$ the inverse of an independent $\alpha$-stable subordinator (see Orsingher and Beghin, 2009). Indeed for $\mathcal{M}(\theta, t):=\mathbb{E} e^{\theta W\left(\mathcal{L}_{\alpha}(t)\right)}$ we have that $\mathcal{M}(\theta, t)=E_{\alpha, 1}\left(\frac{\theta^{2}}{2} t^{\alpha}\right)$.

\section{Further generalizations}

We generalize the results of the previous sections by considering the FokkerPlanck equation (in Fourier space) with a convolution-type operator defined by means of a general Bernstein function (not necessarily fractional).

Definition 4.1. Let $u: \mathbb{R}^{+} \rightarrow \mathbb{R}$ be an absolutely continuous function and $D_{x}^{g}$ be the convolution-type derivative defined in (4), then

$$
\mathcal{L}_{x}^{g} u(x):=u(x) D_{x}^{g} \log u(x), \quad x \in \mathbb{R}^{+} .
$$

We start by proving the following preliminary result which concerns the transition density of the inverse subordinator: let us denote by $\mathcal{A}^{g}(t), t \geq 0$, the subordinator with Laplace exponent $g(s)$, i.e. such that

$$
\mathbb{E} e^{-s \mathcal{A}^{g}(t)}=e^{-g(s) t} .
$$

Let moreover $L^{g}(t), t \geq 0$, be its inverse, i.e.

$$
L^{g}(t)=\inf \left\{s>0: \mathcal{A}^{g}(s)>t\right\}, \quad s, t>0
$$

and $l_{g}(x, t)=\operatorname{Pr}\left\{L^{g}(t) \in d x\right\}$ be its transition density.

Lemma 4.2. The following initial-value problem

$$
D_{t}^{g} u(t)=-k u(t), \quad t, k \geq 0,
$$

with $u(0)=1$, is satisfied by the Laplace transform of the inverse subordinator density $l_{g}(x, t)$, i.e. by

$$
\tilde{l}_{g}(k, t)=\int_{0}^{+\infty} e^{-k x} l_{g}(x, t) d x=\mathbb{E} e^{-k L^{g}(t)} .
$$


Proof: By considering the Laplace transform of the inverse subordinator given in Toaldo (2015, Proposition 3.2), we get

$$
\widetilde{u}(s)=\frac{g(s)}{s} \int_{0}^{+\infty} e^{-k x} e^{-g(s) x} d x=\frac{g(s)}{s} \frac{1}{k+g(s)} .
$$

On the other hand, from (4.2), we get, by (1.6) together with the initial condition,

$$
g(s) \int_{0}^{+\infty} e^{-s t} \widetilde{l}_{g}(k, t) d t-\frac{g(s)}{s}=-k \int_{0}^{+\infty} e^{-s t} \widetilde{l}_{g}(k, t) d t .
$$

By means of the previous result, we can prove the following generalization of Theorem 3.1.

Theorem 4.3. Let $\mathcal{L}_{t}^{g}$ be the operator defined in Def.4.1, then the solution of the generalized Fokker-Planck equation (in Fourier space)

$\mathcal{L}_{t}^{g} \widehat{u}(\xi, t)=-\frac{\gamma}{2^{1-\alpha}} \xi \frac{\partial}{\partial \xi} \widehat{u}(\xi, t)-\frac{\theta}{2^{1-\alpha}} \xi^{2} \widehat{u}(\xi, t), \quad \xi \in \mathbb{R}, t \geq 0, \alpha \in(0,1], D, \gamma>0$,

with initial condition $\widehat{u}(\xi, 0)=1$, for any $t \geq 0$, is given by

$$
\widehat{u}(\xi, t)=\exp \left\{-\frac{\theta \xi^{2}}{2 \gamma}\left[1-\widetilde{l}_{g}(\gamma, t)\right]\right\} .
$$

Proof: By applying Lemma 4.2, we get

$$
\begin{aligned}
D_{t}^{g} \log \widehat{u}(\xi, t) & =-\frac{\theta \xi^{2}}{2 \gamma} D_{t}^{g}\left[1-\widetilde{l}_{g}(\gamma, t)\right] \\
& =-\theta \xi \widetilde{l}_{g}(\gamma, t) \\
& =-2 \gamma \log \widehat{u}(\xi, t)-\theta \xi^{2}
\end{aligned}
$$

and thus

$$
\begin{aligned}
\widehat{u}(\xi, t) D_{t}^{g} \log \widehat{u}(\xi, t) & =\theta \xi^{2}\left[1-\widetilde{l}_{g}(\gamma, t)\right] \widehat{u}(\xi, t)-\theta \xi^{2} \widehat{u}(\xi, t) \\
& =-\xi \gamma \frac{\partial}{\partial \xi} \widehat{u}(\xi, t)-\theta \xi^{2} \widehat{u}(\xi, t),
\end{aligned}
$$

which coincides with (4.4). The initial condition is verified since $\widetilde{l}_{g}(\gamma, 0)=\int_{0}^{+\infty} e^{-\gamma x} l_{g}(x, 0) d x=\int_{0}^{+\infty} e^{-\gamma x} \delta(x) d x=1$.

In order to define the generalized time-changed OU process, we check that $\mathcal{T}_{g}(\cdot):=-\frac{1}{2 \gamma} \log \widetilde{l}_{g}(\gamma, \cdot)$ is an increasing and continuous function. Let us denote by $h_{g}(x, t)$ the density of the subordinator $\mathcal{A}^{g}$, then we get, for any $t>0$,

$$
\begin{aligned}
\widetilde{l}_{g}(\gamma, t) & =\int_{0}^{+\infty} e^{-\gamma x} l_{g}(x, t) d x \\
& =\int_{0}^{+\infty} e^{-\gamma x} \frac{\partial}{\partial x} P\left\{L^{g}(t)<x\right\} d x \\
& =\int_{0}^{+\infty} e^{-\gamma x} \frac{\partial}{\partial x} P\left\{\mathcal{A}^{g}(x)>t\right\} d x \\
& =\left[e^{-\gamma x} P\left\{\mathcal{A}^{g}(x)>t\right\}\right]_{x=0}^{+\infty}+\gamma \int_{0}^{+\infty} e^{-\gamma x} P\left\{\mathcal{A}^{g}(x)>t\right\} d x
\end{aligned}
$$




$$
\begin{aligned}
& =\gamma \int_{0}^{+\infty} e^{-\gamma x}\left(\int_{t}^{+\infty} h_{g}(s, x) d s\right) d x \\
& =\gamma \int_{t}^{+\infty}\left(\int_{0}^{+\infty} e^{-\gamma x} h_{g}(s, x) d x\right) d s,
\end{aligned}
$$

which is clearly decreasing, since the quantity inside brackets is positive. Continuity follows from Proposition 3.2 in Toaldo (2015).

Definition 4.4. Let $\left(\Omega, \mathcal{F}, \mathcal{F}_{t}, P\right)$ be a probability space with filtration $\mathcal{F}_{t}$ and let $\mathcal{X}_{g}:=\left\{\mathcal{X}_{g}(t), t \geq 0\right\}$ be defined as $\mathcal{X}_{g}(t):=X\left(\mathcal{T}_{g}(t)\right)$, where $X$ is the standard OU process and $\mathcal{T}_{g}(t)=-\frac{1}{2 \gamma} \log \widetilde{l}_{g}(\gamma, t)$.

Remark 4.5. It is easy to check that $\mathcal{X}_{g}$ is a Gaussian process, with $\mathbb{E} \mathcal{X}_{g}(t)=0$ and

$$
\operatorname{Cov}\left(\mathcal{X}_{g}(t), \mathcal{X}_{g}(s)\right)=\frac{\theta}{\gamma} \sqrt{\frac{\widetilde{l}_{g}(\gamma, t \vee s)}{\widetilde{l}_{g}(\gamma, t \wedge s)}}\left[1-\widetilde{l}_{g}(\gamma, t \wedge s)\right],
$$

for $t, s \geq 0, \gamma>0$ and for any Bernstein function $g$. Moreover, the process $\mathcal{X}_{g}$ is related to the Brownian motion by the following equality in distribution

$$
\mathcal{X}_{g}(t) \stackrel{d}{=} \sqrt{\frac{\theta}{\gamma} \widetilde{l}_{g}(\gamma, t)} W\left(\frac{1}{\widetilde{l}_{g}(\gamma, t)}-1\right) .
$$

The definition of fractional OU processes given in section 3.2 can be analogously generalized, under the assumption that $\widetilde{l}_{g}(\gamma,|\cdot|)$ is a positive definite function, both in the stationary and non-stationary cases. From (4.6), we can write the following relationship between the derivatives of $\widetilde{l}_{g}(\gamma, t)$ and $\widetilde{h}_{g}(t, \gamma)$ :

$$
\frac{\partial^{r}}{\partial t^{r}} \widetilde{l}_{g}(\gamma, t)=-\gamma \frac{\partial^{r}}{\partial t^{r}} \widetilde{h}_{g}(t, \gamma)
$$

for any $r=1,2, \ldots$. Therefore, by steps similar to Lemma 3.6, we can conclude that $\widetilde{l}_{g}(\gamma,|\cdot|)$ is a positive definite function if $\widetilde{l}_{g}(\gamma, \cdot)$ is CM and (from (4.8)) this holds if the subordinator $\mathcal{A}^{g}$ is such that the time-Laplace transform of its density, i.e. $\widetilde{h}_{g}(\cdot, \gamma)$, is a Bernstein function.

Definition 4.6. Let $\mathcal{A}^{g}$ be a subordinator such that the time-Laplace transform of its density, i.e. $\widetilde{h}_{g}(\cdot, \gamma)$, is a Bernstein function. Let $\overline{\mathcal{Y}}_{g}:=\left\{\overline{\mathcal{Y}}_{g}(t), t \geq 0\right\}$ be a Gaussian process with $\mathbb{E} \overline{\mathcal{Y}}_{g}(t)=0$, for any $t \geq 0$ and

$$
r(s):=\operatorname{Cov}\left(\overline{\mathcal{Y}}_{g}(t), \overline{\mathcal{Y}}_{g}(t+s)\right)=\frac{\theta}{\gamma} \widetilde{l}_{g}(\gamma,|s|),
$$

for any $s \in \mathbb{R}, \gamma>0$.

We can also give a condition for long-range dependence of $\overline{\mathcal{Y}}_{g}$ in terms of the tail's probability of the subordinator, i.e. $P\left\{\mathcal{A}^{g}(x)>t\right\}$, for $t \rightarrow+\infty$. Indeed it is evident from (4.6) that if

$$
P\left\{\mathcal{A}^{g}(x)>t\right\} \sim K_{x} t^{1-H},
$$

for some $H \in(0,1)$ and a constant $K_{x}>0$, then $r(s)=\frac{\theta}{\gamma} \widetilde{l}_{g}(\gamma, s) \sim K_{\gamma}^{\prime} s^{1-H}$ for $s \rightarrow+\infty$ and for $K_{\gamma}^{\prime}>0$. Thus, under condition (4.10), the process is long-memory of order $H$, in the covariance sense. 
Definition 4.7. Let $\overline{\mathcal{Y}}_{g}$ be defined as in Def.4.6 and let

$$
\mathcal{Y}_{g}(t)=\overline{\mathcal{Y}}_{g}(t)-\sqrt{\widetilde{l}_{g}(\gamma, 2 t)} \mathcal{Z}
$$

where $\left(\overline{\mathcal{Y}}_{g}(t), \mathcal{Z}\right)$ is a Gaussian centered random vector with covariance matrix

$$
\sum=\left(\begin{array}{cc}
\frac{\theta}{\gamma} & \frac{\theta}{\gamma} \sqrt{\left.\widetilde{l}_{g}(\gamma, 2 t)\right)} \\
\frac{\theta}{\gamma} \sqrt{\tilde{l}_{g}(\gamma, 2 t)} & \frac{\theta}{\gamma}
\end{array}\right),
$$

for any $t \geq 0$.

Remark 4.8. The generalized OU process $\mathcal{Y}_{g}$ is centered, Gaussian, with

$$
\operatorname{Cov}\left(\mathcal{Y}_{g}(t), \mathcal{Y}_{g}(s)\right)=\frac{\theta}{\gamma}\left[\widetilde{l}_{g}(\gamma,|t-s|)-\sqrt{\widetilde{l}_{g}(\gamma, 2 t) \widetilde{l}_{g}(\gamma, 2 s)}\right],
$$

for $s, t \geq 0$ and $\theta, \gamma>0$. Since $\mathcal{Y}_{g}$ is a zero-mean Gaussian process, its finitedimensional distributions are completely characterized by its autocovariance function. The characteristic function of $\mathcal{Y}_{g}$ coincides with (4.5) and therefore the onedimensional density of the process $\mathcal{Y}_{g}$ coincides with the solution to the generalized FP equation (4.4). Moreover, $\mathcal{Y}_{g}$ is non-Markovian and mean-reverting, since $\lim _{t \rightarrow \infty} \operatorname{Var} \mathcal{Y}_{\alpha}(t)=\lim _{t \rightarrow \infty} \theta / \gamma\left[1-\widetilde{l}_{g}(\gamma, 2 t)\right]=\theta / \gamma$, as can be checked in (4.6).

We now show how the results of sections 3.1 and 3.2 can be derived as special cases and we also present another interesting special case. Other examples could be derived analogously, by specifying the Bernstein function and the corresponding Lévy measure.

Example 4.9. (Stable case) In the special case where $g(s)=s^{\alpha}$, for $\alpha \in(0,1]$, the process $\mathcal{A}^{g}$ coincides with the $\alpha$-stable subordinator with $\mathbb{E} e^{-s \mathcal{A}^{g}(t)}=e^{-s^{\alpha} t}$ (whose Lévy density is $\bar{\nu}(x)=x^{-\alpha-1} / \Gamma(m-\alpha)$ ). Formula (1.5) reduces to the Caputo derivative (1.2), for $m=1$. Thus the operator defined in (4.1) coincides with $\mathcal{L}_{t}^{\alpha}$ given in Def.1.1. It is immediate to check that, in this special case, formula (4.3) coincides with the Laplace transform of the inverse $\alpha$-stable subordinator, i.e. $\widetilde{l}_{\alpha}(\gamma, t)=\int_{0}^{+\infty} e^{-\gamma x} l_{\alpha}(x, t) d x=E_{\alpha, 1}\left(-\gamma t^{\alpha}\right)$ (see Meerschaert and Straka, 2013). Therefore the result of Lemma 4.2 reduces to the well-known property of the MittagLeffler function of being the eigenfunction of the Caputo fractional derivative (see, e.g., Lemma 2.23 in Kilbas et al., 2006). Correspondingly, Def.4.6 reduces to Def.3.7 and formula (4.7) coincides with (3.5).

Example 4.10. (Compound Poisson with exponential jumps) Another interesting case can be obtained by specializing the previous results to the case where $g(s)=s /(s+a)$, with $a>0$. This Bernstein function corresponds to the exponential Lévy density $\bar{\nu}(x)=a e^{-a x}$ (see Schilling et al., 2012, p.304). The density of the inverse subordinator can be obtained explicitely as follows:

$$
l_{g}(x, t)=\int_{0}^{t} h_{g}(z, x) \nu(t-z) d z=\int_{0}^{t} h_{g}(z, x) e^{-a(t-z)} d z,
$$

where the subordinator's density can be evaluated by inverting its Laplace transform:

$$
\widetilde{h}_{g}(\eta, x)=e^{-x \frac{\eta}{\eta+a}}=e^{-x} e^{\frac{x a}{\eta+a}}=e^{-x}+e^{-x} \sum_{j=1}^{\infty} \frac{(x a)^{j}}{j !(\eta+a)^{j}} .
$$


Thus

$$
h_{g}(z, x)=e^{-x} \delta(z)+\frac{e^{-x-a z}}{z} \sum_{j=1}^{\infty} \frac{(x a z)^{j}}{j !(j-1) !}
$$

which is the transition density of the compound Poisson with exponential jumps $\sum_{n=1}^{N(t)} X_{j}, t>0$, where $N(t), t>0$ is the Poisson process with unitary intensity and $X_{j}$ are exponential i.i.d. random variables with parameter $a$ for any $j=1,2, \ldots$ (see Beghin and Macci, 2012, for details). By substituting in (4.12) and by standard calculus, we get

$$
l_{g}(x, t)=e^{-x-a t} \sum_{j=0}^{\infty} \frac{(x a t)^{j}}{(j !)^{2}}=e^{-x-a t} W_{1,1}(x a t)
$$

where $W_{\beta, \gamma}(x)=\sum_{j=0}^{\infty} \frac{x^{\beta j}}{j ! \Gamma(\beta j+\gamma)}, x, \beta, \gamma \in \mathbb{C}$, is the Wright function (see Kilbas et al., 2006, p.54). Therefore $\widetilde{l}_{g}(\gamma, t)$ is given by

$$
\widetilde{l}_{g}(\gamma, t)=\frac{e^{-a t \frac{\gamma}{\gamma+1}}}{\gamma+1}
$$

and the time transform in Def.4.4, i.e. $\mathcal{T}_{g}(t)=\frac{a}{2(\gamma+1)} t+2 \gamma \log (\gamma+1)$, is surprisingly linear in $t$. As a consequence, we can conclude that the generalized Fokker-Planck equation (4.4), with the operator $\mathcal{L}_{t}^{g}$ in Def.4.1 specialized with

$$
D_{t}^{g} u(t)=\int_{0}^{t} \frac{d}{d s} u(t-s) e^{-a s} d s,
$$

is satisfied by the transition density of a standard OU process under a linear combination of the time argument.

Finally, since (4.13) is clearly positive definite, the stationary version of the previous process is the centered Gaussian process with covariance

$$
r(s)=\frac{\theta}{\gamma} \frac{e^{-\frac{a \gamma}{\gamma+1}|s|}}{\gamma+1},
$$

for any $s \in \mathbb{R}, \gamma, \theta>0$.

\section{References}

D. Applebaum. Lévy processes and stochastic calculus, volume 116 of Cambridge Studies in Advanced Mathematics. Cambridge University Press, Cambridge, second edition (2009). ISBN 978-0-521-73865-1. MR2512800.

O. E. Barndorff-Nielsen and N. N. Leonenko. Spectral properties of superpositions of Ornstein-Uhlenbeck type processes. Methodol. Comput. Appl. Probab. 7 (3), 335-352 (2005). MR2210585.

L. Beghin. Fractional diffusion-type equations with exponential and logarithmic differential operators. Stochastic Process. Appl. 128 (7), 2427-2447 (2018). MR3804799.

L. Beghin and C. Macci. Alternative forms of compound fractional Poisson processes. Abstr. Appl. Anal. pages Art. ID 747503, 30 (2012). MR2991021.

P. Cheridito, H. Kawaguchi and M. Maejima. Fractional Ornstein-Uhlenbeck processes. Electron. J. Probab. 8, no. 3, 14 (2003). MR1961165. 
R. Cont and P. Tankov. Financial modelling with jump processes. Chapman \& Hall/CRC Financial Mathematics Series. Chapman \& Hall/CRC, Boca Raton, FL (2004). ISBN 1-5848-8413-4. MR2042661.

J. Gajda and A. Wyłomańska. Time-changed Ornstein-Uhlenbeck process. J. Phys. A 48 (13), 135004, 18 (2015). MR3323360.

R. Gorenflo, A. A. Kilbas, F. Mainardi and S. V. Rogosin. Mittag-Leffler functions, related topics and applications. Springer Monographs in Mathematics. Springer, Heidelberg (2014). ISBN 978-3-662-43929-6; 978-3-662-43930-2. MR3244285.

H. J. Haubold, A. M. Mathai and R. K. Saxena. Mittag-Leffler functions and their applications. J. Appl. Math. pages Art. ID 298628, 51 (2011). MR2800586.

L. Heinrich and V. Schmidt. Normal convergence of multidimensional shot noise and rates of this convergence. Adv. in Appl. Probab. 17 (4), 709-730 (1985). MR809427.

J. Janczura, S. Orzełand A. Wyłomańska. Subordinated $\alpha$-stable ornstein-uhlenbeck process as a tool for financial data description. Physica A: Statistical Mechanics and its Applications 390 (23), 4379 - 4387 (2011). DOI: 10.1016/j.physa.2011.07.007.

J. H. Jeon, A. V. Chechkin and R. Metzler. Scaled Brownian motion: a paradoxical process with a time dependent diffusivity for the description of anomalous diffusion. Phys Chem Chem Phys 16 (30), 15811-7 (2014). DOI: 10.1039/C4CP02019G.

T. Kaarakka and P. Salminen. On fractional Ornstein-Uhlenbeck processes. Commun. Stoch. Anal. 5 (1), 121-133 (2011). MR2808539.

A. A. Kilbas, H. M. Srivastava and J. J. Trujillo. Theory and applications of fractional differential equations, volume 204 of North-Holland Mathematics Studies. Elsevier Science B.V., Amsterdam (2006). ISBN 978-0-444-51832-3; 0-444-518320 . MR2218073.

N. N. Leonenko, M. M. Meerschaert and A. Sikorskii. Correlation structure of fractional Pearson diffusions. Comput. Math. Appl. 66 (5), 737-745 (2013). MR3089382.

S. G. Lim and S. V. Muniandy. Self-similar Gaussian processes for modeling anomalous diffusion. Phys. Rev. E 66, 021114 (2002). DOI: 10.1103/PhysRevE.66.021114.

M. Magdziarz. Fractional Ornstein-Uhlenbeck processes. Joseph effect in models with infinite variance. Physica A: Statistical Mechanics and its Applications 387 (1), 123-133 (2008). DOI: 10.1016/j.physa.2007.08.016.

M. Magdziarz. Stochastic representation of subdiffusion processes with timedependent drift. Stochastic Process. Appl. 119 (10), 3238-3252 (2009). MR2568272.

M. Magdziarz, A. Weron and K. Weron. Fractional fokker-planck dynamics: Stochastic representation and computer simulation. Phys. Rev. E 75, 016708 (2007). DOI: 10.1103/PhysRevE.75.016708.

F. Mainardi. On some properties of the Mittag-Leffler function $E_{\alpha}\left(-t^{\alpha}\right)$, completely monotone for $t>0$ with $0<\alpha<1$. Discrete Contin. Dyn. Syst. Ser. B 19 (7), 2267-2278 (2014). MR3253257.

A. M. Mathai, R. K. Saxena and H. J. Haubold. The H-function. Theory and applications. Springer, New York (2010). ISBN 978-1-4419-0915-2. MR2562766. 
M. M. Meerschaert and A. Sikorskii. Stochastic models for fractional calculus, volume 43 of De Gruyter Studies in Mathematics. Walter de Gruyter \& Co., Berlin (2012). ISBN 978-3-11-025869-1. MR2884383.

M. M. Meerschaert and P. Straka. Inverse stable subordinators. Math. Model. Nat. Phenom. 8 (2), 1-16 (2013). MR3049524.

A. Mura, M. S. Taqqu and F. Mainardi. Non-Markovian diffusion equations and processes: analysis and simulations. Phys. A 387 (21), 5033-5064 (2008). MR2588003.

E. Orsingher and L. Beghin. Fractional diffusion equations and processes with randomly varying time. Ann. Probab. 37 (1), 206-249 (2009). MR2489164.

A. Papoulis. High density shot noise and Guassianity. J. Appl. Probability 8, 118-127 (1971). MR0278412.

A. Papoulis. Probability, random variables, and stochastic processes. McGraw-Hill Book Co., third edition (1991).

G. A. Pavliotis. Stochastic processes and applications. Diffusion processes, the Fokker-Planck and Langevin equations, volume 60 of Texts in Applied Mathematics. Springer, New York (2014). ISBN 978-1-4939-1322-0; 978-1-4939-1323-7. MR3288096.

D. Revuz and M. Yor. Continuous martingales and Brownian motion, volume 293 of Grundlehren der Mathematischen Wissenschaften [Fundamental Principles of Mathematical Sciences]. Springer-Verlag, Berlin, third edition (1999). ISBN 3540-64325-7. MR1725357.

K. Sato. Lévy processes and infinitely divisible distributions, volume 68 of Cambridge Studies in Advanced Mathematics. Cambridge University Press, Cambridge (1999). ISBN 0-521-55302-4. MR1739520.

R. L. Schilling, R. Song and Z. Vondraček. Bernstein functions. Theory and applications, volume 37 of De Gruyter Studies in Mathematics. Walter de Gruyter \& Co., Berlin, second edition (2012). ISBN 978-3-11-025229-3; 978-3-11-026933-8. MR2978140.

W. R. Schneider. Completely monotone generalized Mittag-Leffler functions. Exposition. Math. 14 (1), 3-16 (1996). MR1382012.

B. Toaldo. Convolution-type derivatives, hitting-times of subordinators and timechanged $C_{0}$-semigroups. Potential Anal. 42 (1), 115-140 (2015). MR3297989.

P. Vellaisamy and K. K. Kataria. The $I$-function distribution and its extensions. Teor. Veroyatn. Primen. 63 (2), 284-305 (2018). Reprinted in Theory Probab. Appl. 63 (2018), no. 2, 227-245. MR3796490. 